\title{
Thermoelectric Effects in Planar Tunnel Junctions
}

M. WILCZYŃSKI

\author{
Faculty of Physics, Warsaw University of Technology, Koszykowa 75, 00-662 Warsaw, Poland
}

\begin{abstract}
The thermopower and the charge current generated by the finite temperature gradient applied to ferromagnetic planar tunnel junctions are investigated in the spin-polarized free-electron-like one-band model. It has been shown that the current depends almost linearly on the temperature difference between the electrodes while the thermopower does not depend significantly on the temperature gradient. The studied quantities depend on the magnetic configuration of the junction. The form of this dependence is sensitive to the height of the barrier, but is not sensitive to the temperature difference between the electrodes.
\end{abstract}

PACS: 72.20.Pa, 73.40.Gk, 73.40.Rw

\section{Introduction}

Thermoelectric effects connected with the relation between the charge transport and temperature gradients have recently attracted much attention [1]. Especially the Seebeck effect related to the bias voltage generated by the temperature gradient and the Peltier effect connected with the temperature gradient caused by the charge transport are widely studied. The investigations are concentrated mainly on the bulk materials, however the nanostructures with quantum dots (e.g. [2,3]) or heterostructures (e.g. Refs. $[4,5]$ ) have attracted more attention due to the hope that in such systems thermoelectric effects are more significant and it will be possible to use them to construct devices of higher efficiency. Slightly less attention is paid to the systems with tunnel barriers. Recent paper concerning the Au nanowires indicates the fact that the tunnel effect can lead to the increase of the thermopower [6]. The papers concerning thermoelectric effects in tunnel junctions are devoted mainly to nonmagnetic systems [6, 7], however tunnel junctions with ferromagnetic electrodes are also studied [8]. The increase of interest in the magnetic systems can be related to new thermoelectric effects related to the spin of electron, e.g. spin Seebeck effect which can appear only in magnetic materials [9].

In the present paper thermoelectric phenomena in planar single tunnel junctions consisting of two ferromagnetic electrodes separated by the tunnel barrier are analyzed. The calculations of the current flowing through the system and the thermopower in junctions with significant difference between the temperatures of the electrodes are performed. The influence of average temperature of the junction and orientation of magnetic moments on these quantities is also studied.

\section{Model}

All calculations are performed in the spin-polarized free-electron-like one-band model. The electronic structure of both electrodes is modeled by the spin-split parabolic band with the electron mass $m$ equal to the mass of the free electron. It is assumed that the electron energy $E$ and the component of the electron wave vector parallel to the electrode/barrier interface $k_{\|}$are conserved during the tunneling. As a result of this, also the energy $\varepsilon_{\perp}=E-\frac{\hbar^{2} k_{\|}^{2}}{2 m}$ connected with the electron motion in the direction perpendicular to the electrode/ barrier interface is conserved and the transmission coefficient can be treated as a function of this energy. To describe the electron wave function, the combination of plane waves is used in the electrodes and exponential functions in the barrier [10]. When the voltage drop across the junction appears, then the exponential functions should be replaced by the Airy functions. The arguments of these functions depend, e.g. on the energy $\varepsilon_{\perp}$. Transmission coefficients are calculated in the usual manner taking into account the continuity conditions of the electron wave functions and their spatial derivatives at the electrode/barrier interfaces. Due to the fact that the magnetic moments in the electrodes form the arbitrary angle $\theta$, in writing the matching conditions at the barrier/right electrode interface the appropriate spinor transformations have to be applied [10]. The charge current flowing through the junction can be calculated from the formula [7]:

$$
\begin{aligned}
I & =\frac{2 \pi m e k}{h^{3}} \sum_{\sigma} \int \mathrm{d} \varepsilon_{\perp} W_{\sigma}\left(\varepsilon_{\perp}\right) \\
& \times\left\{T_{\mathrm{L}} \ln \left[1+\exp \left(\frac{E_{f}-\varepsilon_{\perp}-e V}{k T_{\mathrm{L}}}\right)\right]\right. \\
& \left.-T_{\mathrm{R}} \ln \left[1+\exp \left(\frac{E_{f}-\varepsilon_{\perp}}{k T_{\mathrm{R}}}\right)\right]\right\} .
\end{aligned}
$$

In the above formula $k$ is the Boltzmann constant, $V$ denotes the voltage drop across the junction, whereas $T_{\mathrm{L}}\left(T_{\mathrm{R}}\right)$ denotes the temperature of the left (right) electrode; $W_{\sigma}\left(\varepsilon_{\perp}\right)$ is the transmission coefficient for the tunneling electron of specified energy $\varepsilon_{\perp}$ and spin $\sigma$ in the left electrode. $W_{\sigma}\left(\varepsilon_{\perp}\right)$ depends on the orientation of magnetic moments in the electrodes. Formula (1) can be used to calculate the tunneling current in a general situation when the temperatures of both electrodes are 
not equal and the finite bias voltage is applied to the junction. Normally in the absence of the bias voltage the current flows through the tunnel junction joined to the closed circuit between the warmer to the colder electrode. However, when the bias voltage of appropriate polarization is applied to the junction the intensity of this current can be reduced to zero. Such bias voltage is also generated in the junction not joined to the closed circuit in which the current does not flow through the junction. This effect is known as the Seebeck effect and can be characterized by the thermopower $S$ calculated from the formula

$$
S=\frac{\mathrm{d} V}{\mathrm{~d} \Delta T} .
$$

In the above formula $V$ denotes the bias voltage generated in the junction in which the temperature difference between electrodes is equal to $\Delta T$.

\section{Results}

In the present paper the charge current $I$ flowing through the junction as a result of the temperature gradient applied to the junction when the bias voltage is not applied is analyzed. In the calculations it is assumed that the average temperature of the junction $T_{\mathrm{av}}=\left(T_{\mathrm{L}}+T_{\mathrm{R}}\right) / 2$ is constant, whereas the temperature difference between the electrodes $\Delta T=T_{\mathrm{L}}-T_{\mathrm{R}}$ is varied. The bias voltage $V$ generated across the junction, when the junction is not joined to the closed circuit and the current does not flow is also determined as a function of $\Delta T$. Additionally, the thermopower is calculated from Eq. (2) while the conductance in the absence of the bias voltage is obtained using the expression

$$
Q_{\mathrm{t}}=\frac{\mathrm{d} I}{\mathrm{~d} \Delta T} \text {. }
$$

Thermopower $S$ and conductance $Q_{\mathrm{t}}$ can generally depend on $\Delta T$, however, as will be shown, this dependence is rather weak.

Most of the calculations are performed for the following parameters: the spin-splitting of the electron bands in the ferromagnetic electrodes $2 \Delta=4.5 \mathrm{eV}$, the Fermi energy measured from the middle point between the bottoms of the spin-split electron bands $E_{f}=2.6 \mathrm{eV}$, the thickness of the barrier $d=1.5 \mathrm{~nm}$. They are conducted for different average temperatures of the junction and for different relative orientations of magnetic moments in the electrodes. The junctions with high and low tunnel barrier are considered.

In Figs. 1 and 2, $I$ and $V$ as a function of $\Delta T$ for junctions with the barrier of height $U=1.5 \mathrm{eV}$ and $U=0.1 \mathrm{eV}$ are presented. As can be seen, both $I$ and $V$ depend almost linearly on $\Delta T$, when $\Delta T$ is not very large. As a result of this, thermopower $S$ and conductance $Q_{\mathrm{t}}$ practically do not depend on $\Delta T$. However, $S$ and $Q_{\mathrm{t}}$ strictly depend on the average temperature of the junction and increase with $T_{\mathrm{av}}$ for all studied magnetic configurations and for both heights of the barrier, which

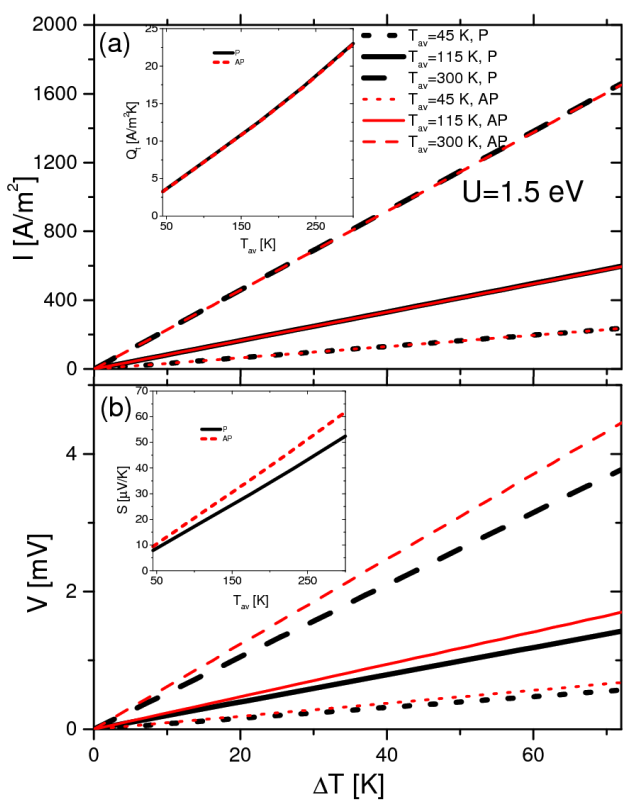

Fig. 1. The current in the closed circuit (a) and the bias voltage in the open circuit (b) as a function of the temperature difference between the electrodes calculated in the junction with barrier of height $U=1.5 \mathrm{eV}$ for different average temperatures of the junction $T_{\mathrm{av}}$ and for the $\mathrm{P}$ and $\mathrm{AP}$ orientation of magnetic moments in the electrodes. The other parameters are: the Fermi energy $E_{f}=2.6 \mathrm{eV}$, the spin-splitting of the electron bands $2 \Delta=4.5 \mathrm{eV}$, the thickness of the barrier $d=1.5 \mathrm{~nm}$. In the insets conductance $Q_{\mathrm{t}}$ and thermopower $S$ obtained for small $\Delta T$ are presented as a function of average temperature $T_{\mathrm{av}}$ of the junction.

can be seen in the insets. They also depend on the magnetic configuration. The form of this dependence in the case of thermopower varies qualitatively with the height of the barrier. $S$ is usually higher in the antiparallel $(\mathrm{AP}, \theta=\pi)$ configuration than in the parallel $(\mathrm{P}, \theta=0)$ configuration, however in the junctions with lower barriers, e.g. with height $U=0.1 \mathrm{eV}$ presented in Fig. $2 \mathrm{~b}$ this dependence can be reversed. $Q_{\mathrm{t}}$ is always higher in the $\mathrm{P}$ than in the AP configuration, however in the junctions of height $U=1.5 \mathrm{eV}$ this dependence is very weak (the curves obtained for $\mathrm{P}$ and AP configurations are almost inseparable in Fig. 1a). This is connected with the fact that in the junctions with barriers of this height $U=1.5 \mathrm{eV}$, the electrons of energy slightly larger than the Fermi energy which make a dominant contribution to the current generated by the temperature gradient have very low effective spin polarization [10]. This polarization is higher for electrons of lower energy mediating the current generated by the bias voltage, which leads to the stronger dependence of the charge current generated by the bias voltage (not shown) and the thermopower on the magnetic configuration. The values of $S$ and $Q_{\mathrm{t}}$ obtained using the method described in the present paper are in quantitative agreement with those obtained using 


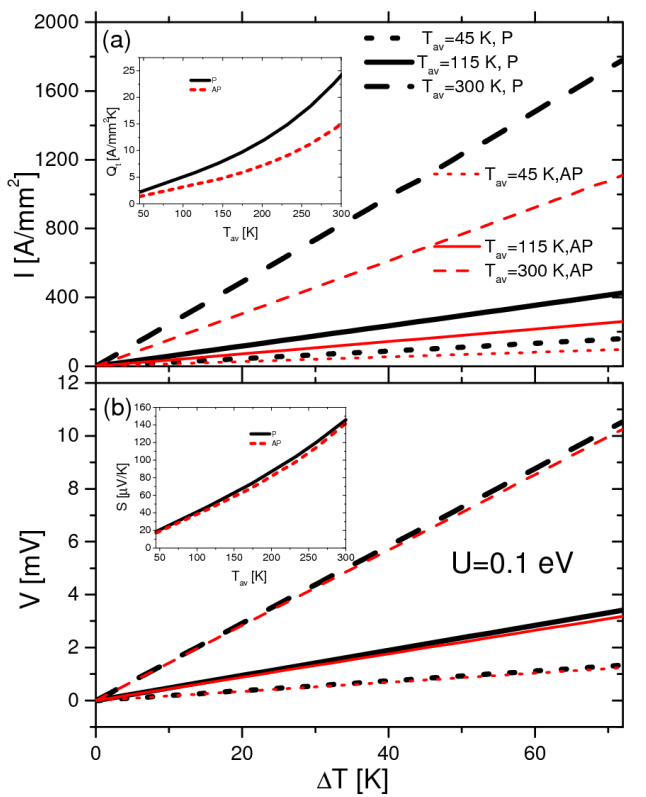

Fig. 2. The current in the closed circuit (a) and the bias voltage in the open circuit (b) as a function of the temperature difference between the electrodes calculated in the junction with barrier of height $U=0.1 \mathrm{eV}$ for different average temperatures of the junction $T_{\mathrm{av}}$ and for the $\mathrm{P}$ and AP orientation of magnetic moments in the electrodes. The other parameters are the same as in Fig. 1. In the insets conductance $Q_{\mathrm{t}}$ and thermopower $S$ obtained for small $\Delta T$ are presented as a function of average temperature $T_{\mathrm{av}}$ of the junction.

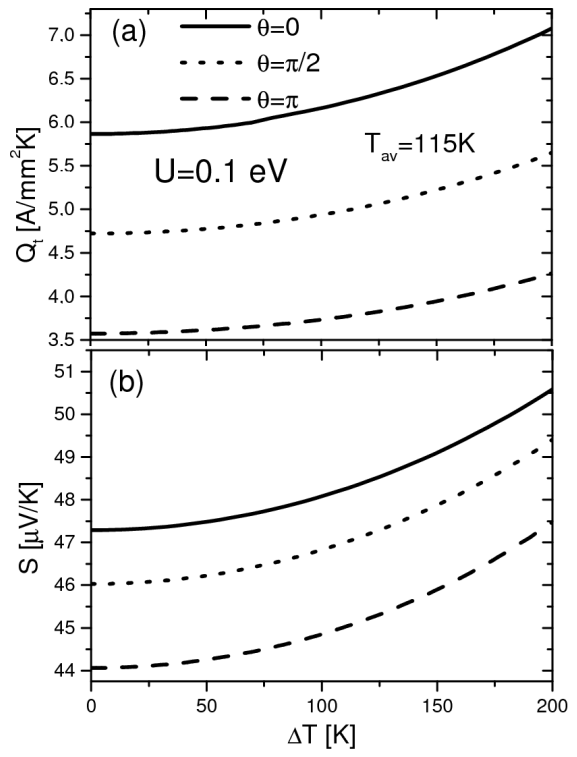

Fig. 3. Conductance $Q_{\mathrm{t}}(\mathrm{a})$ and thermopower $S(\mathrm{~b})$ as a function of the difference between the temperatures of the electrodes in the junction with barrier of height $U=$ $0.1 \mathrm{eV}$ calculated for different angles $\theta$ formed by the magnetic moments in the electrodes and for the average temperature $T_{\mathrm{av}}=115 \mathrm{~K}$. The other parameters are the same as in Fig. 1. the linear response theory [3]. The significant discrepancy appears only when the large temperature gradient is applied to the junction and the barrier is low. It can be seen in Fig. 3 where $S$ and $Q_{\mathrm{t}}$ versus $\Delta T$ for $U=0.1 \mathrm{eV}$, $T_{\mathrm{av}}=115 \mathrm{~K}$ and for the parallel $(\theta=0)$, antiparallel $(\theta=\pi)$ and perpendicular $(\theta=\pi / 2)$ magnetic configuration are presented. In this figure the increase of $S$ and $Q_{\mathrm{t}}$ with $\Delta T$ can be observed, which can be related to the fact that for such large $\Delta T$ the dependence of the current and the bias on $\Delta T$ deviates more significantly from linear dependence than in the case when $\Delta T$ is not very large. However, in the junction with large $\Delta T$ the character of the dependence of $S$ and $Q_{\mathrm{t}}$ on the magnetic configuration is not changed. It can be noticed that the results obtained correspond to the situation when the average temperature of the junction is constant so the temperature of the left electrode is increased, while the temperature of the right electrode is reduced. In the situation when the temperature of one of the two electrodes is kept constant and only the temperature of the second electrode is increased, the current $I$ and bias $V$ do not depend linearly on $\Delta T$ and $S$ and $Q_{\mathrm{t}}$ increase significantly with $\Delta T$. This increase can be related to the increase of the average temperature of the junctions, which leads to the additional significant increase of $S$ and $Q_{\mathrm{t}}$.

\section{References}

[1] F. Giazotto, T.T. Heikkilä, A. Luukanen, A.M. Savin, J.P. Pekola, Rev. Mod. Phys. 78, 217 (2006).

[2] C.W.J. Beenakker, A.A.M. Staring, Phys. Rev. B 46, 9667 (1992).

[3] R. Świrkowicz, M. Wierzbicki, J. Barnaś, Phys. Rev. B 80, 195409 (2009).

[4] R. Venkatasubramanian, E. Siivola, T. Colpitts, B. O'Quinn, Nature 413, 597 (2001).

[5] M. Hatami, G.E.W. Bauer, Q. Zhang, P.J. Kelly, Phys. Rev. B 79, 174426 (2009).

[6] N.B. Duarte, G.D. Mahan, S. Tadigadapa, Nano Lett. 9, 617 (2009).

[7] J. Marshall, A. Majumdar, J. Appl. Phys. 74, 4000 (1993).

[8] Z.-C. Wang, G. Su, S. Gao, Phys. Rev. B 63, 224419 (2001).

[9] K. Uchida, S. Takahashi, K. Harii, J. Ieda, W. Koshibae, K. Ando, S. Maekawa, E. Saitoh, $\mathrm{Na}$ ture 455, 778 (2008).

[10] J.C. Slonczewski, Phys. Rev. B 39, 6995 (1989). 